American Journal of Pharmacology and Toxicology 2 (2): 98-105, 2007

ISSN 1557-4962

(C) 2007 Science Publications

\title{
Insertion of poly (ethylene glycol)-lipid reduces the liposome-encapsulated hemoglobin- induced thrombocytopenic reaction
}

\author{
${ }^{1,2}$ Vibhudutta Awasthi, ${ }^{1}$ Beth Goins and ${ }^{1}$ William T. Phillips \\ ${ }^{1}$ Department of Radiology, University of Texas Health Science Center at San Antonio, \\ 7703 Floyd Curl Drive, San Antonio, TX, USA 78229-3900. \\ ${ }^{2}$ Current affiliation is Department of Pharmaceutical Sciences, College of Pharmacy, \\ 1110 N. Stonewall Avenue, Oklahoma City, OK, USA 73117.
}

\begin{abstract}
Interaction with platelets has been recognized as a concern in the development of liposomeencapsulated hemoglobin as an oxygen carrier. This reaction causes a significant drop in circulating platelets in a rapid and transient fashion. We studied the effect of lipid bilayer charge on the magnitude of drop in circulating platelets. We also investigated the effect of post-inserted PEG-lipid on this phenomenon. PEG-DSPE was inserted in the outer lipid layer of neutral and anionic LEHs. Autologous rabbit platelets were radiolabeled with In-111-oxime and allowed to circulate for $30 \mathrm{~min}$ before administering a small dose of LEH preparation. Circulating radioactivity as well as the number of platelets was monitored by sampling blood at various time points. Anionic LEH induced the largest decrease $(64 \%)$ in circulating platelets. PEGylation of anionic LEH significantly inhibited thrombocytopenic reaction $(45.3 \%$ decrease). PEGylated neutral LEH demonstrated the least thrombocytopenia (23.8\% decrease). In all cases, the reaction was transient, and the platelet counts recovered to reach the baseline level. The recovery time, however, was dependent on the charge and surface PEGylation of LEH preparations. The results indicate that the platelet reaction is significantly influenced by the surface charge carried by the liposomal surface. Hiding the charge by surface PEGylation appears to be an effective means of reducing the thrombocytopenic reaction shown by the LEH.
\end{abstract}

Key words: Liposome-encapsulated hemoglobin, thrombocytopenia, platelet, Poly(ethylene glycol)

\section{INTRODUCTION}

Liposome-encapsulated hemoglobin (LEH) is a novel formulation which is being developed as an oxygen carrier to replace autologous blood transfusions $^{[1,2]}$. A primary requirement for a successful LEH preparation is that it should circulate in blood for a prolonged period while being capable of transporting oxygen. In addition, it is imperative that LEH does not interact adversely with the circulating cellular elements. LEH infusion has previously been shown to induce thrombocytopenia in animal models ${ }^{[3]}$. Such an undesired reaction remains an obstacle to the successful application of LEH as a universal resuscitation fluid. The observation that in vitro platelet aggregation is not affected by incubation with LEH indicates an essential role of a systemic element in inducing platelet reaction $^{[4]}$. It has been since demonstrated that complement system plays an integral role in LEHinduced thrombocytopenia ${ }^{[5]}$ causing activation of classical complement pathway after interaction between the phospholipid bilayer and $\mathrm{C}$ reactive protein. The length of diacyl chain in the phospholipid influences the efficacy of this interaction with DMPC more effective than DPPC or DSPC ${ }^{[6]}$. Complement activation may also occur due to the presence of anti-phospholipid antibodies in circulation ${ }^{[7]}$. The molecular basis of this activation has not been yet established, but it appears that intracellular protein phosphorylation is one of the initial and mandatory steps in the process that is partially dependent on cyclic adenosine monophosphate ${ }^{[8]}$. A recent article has also shown that anionic charge on the surface of the liposome plays a key role in activation of both classical and alternative complement pathway ${ }^{[9]}$.

The extent of thrombocytopenia induced by the liposomes and the time for thrombocyte recovery depends upon the rapidity of administration, the amount administered, composition of liposomes and any associated impurity. In general, a bolus injection has a

Corresponding Author: VD Awasthi, Department of Pharmaceutical Sciences, College of Pharmacy, 1110 N. Stonewall Avenue, Oklahoma City, OK, USA 73117. Phone: (405) 271 6593, Fax: (405) 2717505 
more severe effect than a slow infusion of liposome preparation. However, the lipid composition of liposomes overrides other factors in regards to the degree of thrombocytopenia observed. Liposomes containing negatively charged (anionic) lipids induce a very rapid decline in circulating platelets that is more severe, and that takes a longer time to recover to normal levels as compared to the liposomes consisting of neutral lipids. Phosphatidylglycerol containing liposomes have been shown to form micro-aggregates with platelets in vitro and it has been suggested that the sequestration of these micro-aggregates by the reticuloendothelial system (RES) causes the decline in circulating platelets ${ }^{[10]}$.

Utility of LEH is most obvious when there is reduction in the oxygen carrying capacity secondary to a severe blood loss. Uncontrolled hemorrhage is also characterized by progressive depletion of circulating thrombocytes resulting in an inability to initiate effective hemostasis. In these circumstances, a further thrombocytopenic reaction to resuscitative fluid would exacerbate an already compromised hemostasis condition. Therefore, it is clear that the LEH preparation should be formulated to eliminate the platelet reaction seen with liposome administration. Surface modification of LEH with poly(ethylene glycol) or PEG is an attractive approach to hide the liposomes by imparting steric hydrophilicity to the liposome surface ${ }^{[11]}$. PEGylation makes liposomes more compatible in vivo, and helps evade opsonization and RES uptake. The resultant "sterically stabilized liposomes" circulate in blood for a prolonged time period $^{[12]}$. In this work, we studied the effect of postinsertion of PEG-lipid in the outer phospholipid layer of neutral and negatively charged LEH on the circulation of rabbit platelets in vivo. The results indicate that the PEG-modification attenuates the charge-dependent thrombocytopenia observed with the LEH.

\section{MATERIALS AND METHODS}

The phospholipids, distearoylphosphatidylcholine (DSPC), and poly(ethyleneglycol) ${ }_{5000}$-distearoylphosphatidylethanolamine ( $\mathrm{PEG}_{5000}$-DSPE) were obtained from Avanti Polar Lipids (Pelham, AL). Cholesterol (Chol) was purchased from Calbiochem (La Jolla, CA) and $\alpha$-tocopherol was purchased from Aldrich (Waukegan, IL). Glutathione (GSH), octyl- $\beta$ glucoside (OBG), and pyridoxal-5' phosphate (PLP) were from Sigma (St. Louis, MO). The radiopharmaceutical, ${ }^{111}$ In-oxine, was obtained commercially (GE Healthcare Nuclear Pharmacy, San
Antonio, TX). For animal experiments, anesthetics xylazine and ketamine were from Phoenix Scientific, Inc. (St. Joseph, MO) and Fort Dodge Animal Health (Fort Dodge, IA), respectively.

Hemoglobin: Frozen human stroma-free oxyhemoglobin $\left(\mathrm{O}_{2}-\mathrm{Hb}\right)$ was a kind gift from US Army, Walter Reed Army Institute of Research- Biological Resources Division (Washington DC). Since carbonylhemoglobin $(\mathrm{CO}-\mathrm{Hb})$ is more stable than $\mathrm{O}_{2}-\mathrm{Hb}$ and can tolerate processing at elevated temperature $\left(\sim 55^{\circ} \mathrm{C}\right)$ and shear, $\mathrm{CO}-\mathrm{Hb}$ was used in LEH manufacturing. Immediately after thawing, $\mathrm{O}_{2}-\mathrm{Hb}$ was carbonylated with carbon monoxide (CO) under aseptic conditions ${ }^{[12,}$ 13].

Preparation of LEH: The method of LEH manufacturing, including PEGylation by post-insertion of PEG-DSPE has been reported elsewhere ${ }^{[1,}$ 12]. Briefly, the lipid phase was homogenized in a microfluidizer (M110-T, Microfluidics Corp., Newton, MA) with hemoglobin containing pyridoxal-5' phosphate as an allosteric modifier. The homogenate containing encapsulated hemoglobin was separated from free hemoglobin by multi-step tangential ultrafiltration. After filtration, the neutral and negative LEH preparations were each divided into two equal halves. One half of each preparation was PEGylated by post-insertion of PEG-DSPE, while the other half was further processed without PEGylation. The compositions of the four types of LEHs were-
A. Neutral LEH (DSPC/Cholesterol/ $\alpha$-tocopherol, 51.4:46.4:2.2)
B. PEG-Neutral LEH with post-inserted PEG- DSPE (DSPC/Cholesterol/ $\alpha$-tocopherol, 51.4:46.4:2.2)-PEG-DSPE
C. Anionic LEH (DSPC/Chol/DMPG/ $\alpha-$ tocopherol, 46:42:9.8:2.2)
D. PEG-Anionic LEH with post-inserted PEG- DSPE (DSPC/Chol/DMPG/ $/$-tocopherol, 46:42:9.8:2.2)-PEG-DSPE

Characterization of LEH: The phospholipid concentration of the LEH was determined by the method of Stewart ${ }^{[14]}$. The oxygen affinity (p50) was measured in a Hemox-analyzer (TCS Scientific Corp., New Hope, PA). Amount of encapsulated hemoglobin was determined by monitoring absorbance of the OBG lysate of LEH at $540 \mathrm{~nm}^{[15]}$. Methemoglobin content was measured in hemoglobin as well as $\mathrm{LEH}^{[16]}$. The particle size was determined by photon correlation spectroscopy using a Brookhaven particle size analyzer 
equipped with argon laser, BI-9000AT digital correlator and BI-200SM goniometer (Holtsville, NY). Each sample was sized for 2 min with detector at $90^{\circ}$ angle and sample housed in a $25^{\circ} \mathrm{C}$ bath. The data was analyzed by non-negatively constrained least squares (CONTIN) using dynamic light scattering software9KDLSW, beta version 1.24 supplied with the instrument. The characteristics of the four preparations are summarized in table 1.

Indium-111 radiolabeling of rabbits platelets: The animal experiments were performed according to the NIH Animal Use and Care Guidelines and were approved by the Institutional Animal Care and Use Committee of the University of Texas Health Science Center at San Antonio. New Zealand white Rabbits (2.5 $\mathrm{Kg}$ ) were obtained from Diamond B, Inc. (San Antonio, TX). The animals were provided food and water $a d$ libitum and allowed to acclimatize for at least a week before performing the study. On the day of the study, the rabbits were anesthetized with ketamine/xylazine cocktail (50 and $10 \mathrm{mg} / \mathrm{Kg}$ body weight, respectively). A central ear artery was catheterized with an $18 \mathrm{G}$ angiocath, and about $40 \mathrm{ml}$ of arterial blood was withdrawn into a tube containing $5 \mathrm{ml}$ of acid-citratedextrose solution (Abbot Laboratories, Abbott Park, Illinois). The blood was gently processed to separate pure population of plasma-free platelets. Briefly, the blood was centrifuged at $150 \mathrm{~g}$ for $15 \mathrm{~min}$ to separate platelet rich plasma (PRP). PRP was spun at $800 \mathrm{~g}$ for $10 \mathrm{~min}$ to obtain platelet poor plasma (PPP) and a platelet button. Several centrifugation steps were required to obtain a pure population of rabbit platelets. The platelets were washed with $2 \mathrm{ml}$ of modified Tyrode's solution (MTS) containing $19 \mathrm{mM} \mathrm{NaOH}, 6.4$ $\mathrm{mM}$ citric acid monohydrate, $137 \mathrm{mM} \mathrm{NaCl}, 2.7 \mathrm{mM}$ $\mathrm{KCl}, 2.2 \mathrm{mM} \mathrm{MgCl} 2.6 \mathrm{H}_{2} \mathrm{O}$ and $1.09 \mathrm{mM}$ glucose and resuspended in about $2 \mathrm{ml}$ of MTS. The recovered platelets were labeled with In-111-oxine, while the separated red blood cell fraction was intravenously reinfused into the animal $(1 \mathrm{ml} / \mathrm{min})$. To radiolabel, 1.5 $\mathrm{ml}$ of In-111-oxine (100-200 $\mu \mathrm{Ci})$ was added drop-wise to the platelet suspension while gently swirling the tube. The mixture was incubated for $30-45 \mathrm{~min}$ at room temperature before spinning at $800 \mathrm{~g}$ for $10 \mathrm{~min}$. The labeled platelets were washed twice with PPP to separate any unincorporated radioactivity. The final platelet preparation was resuspended in $3 \mathrm{ml}$ of PPP for injection. The labeling efficiency routinely ranged from $40-70 \%$. As a quality control, randomly selected labeled platelet preparations were microscopically observed for aggregation response to addition of calcium ions.

Animal Study: Rabbits were anesthetized with ketamine/xylazine cocktail. Autologous In-111-platelets (30-70 $\mu \mathrm{Ci})$ were infused over $2 \mathrm{~min}$ via marginal ear vein of the rabbits. Since it has been reported that radiolabeled platelets may be transiently sequestered by liver ${ }^{[17]}$, the platelets were allowed to circulate for 30 min and attain an equilibrium blood level. Blood samples $(0.3 \mathrm{ml})$ were collected at 10,20 and 30 minutes after the platelet infusion to ensure equilibrium circulation level of radiolabeled platelets. A small dose of LEH preparation ( $1 \mathrm{ml}$, about $10 \mathrm{mg}$ phospholipid) was injected intravenously over a period of $1 \mathrm{~min}$. Arterial blood samples $(0.3 \mathrm{ml})$ were withdrawn via an arterial catheter at $0,1,3,5,10,15,20,30,45,60$ and 90 minutes after LEH injection. Control rabbits were similarly treated, but were injected with $3 \mathrm{ml}$ of saline in place of LEH. The collected blood samples were immediately divided into two portions- a $0.1 \mathrm{ml}$ aliquot was taken for radioactivity counting (Wizard 1480, Perkin Elmer Life Sciences, Boston, MA) while the remaining $0.2 \mathrm{ml}$ of the blood was anti-coagulated with $0.8 \mathrm{ml}$ of acid-citrate-dextrose solution. The anticoagulated portion was kept on ice and sent for automated complete blood cell counting. At the end of the study the animals were euthanized.

Data Analysis: All average values are given as \pm standard error of mean. The amount of circulating radioactivity (or the number of platelets) at any given time point was expressed as a percent of the baseline circulating radioactivity (or the number of platelets). The baseline circulating radioactivity (or the number of platelets) was the radioactivity (or the number of platelets) in the blood sample at zero time (Figure 2 and 3). The data was statistically analyzed by the analysis of variance using Prism software for Windows (GraphPad, San Diego, CA). The acceptable probability for significance was $\mathrm{p}<0.05$.

\section{RESULTS}

We manufactured four preparations of LEH (Table 1). The post-inserted PEG within the outer lipid layer was estimated to be around $50-58 \%$ of the added PEG-lipid. Upon storage $4^{\circ} \mathrm{C}$, the PEGylated preparations showed dramatic reduction in sedimentation rate and remained homogenously suspended for at least 3 months. Anionic preparations were also found to be physically stable. On the other hand, neutral LEH without PEG coating was observed to sediment accompanied by an increase in size and non-homogeneity. 
Table 1: Characteristics of LEH preparations

\begin{tabular}{lllll}
\hline & $\begin{array}{l}\text { Size } \\
(\mathrm{nm} \pm \mathrm{sem})\end{array}$ & $\begin{array}{l}{[\mathrm{Lipid}]} \\
(\mathrm{mg} / \mathrm{ml})\end{array}$ & $\begin{array}{l}\mathrm{p} 50 \\
(\mathrm{~mm} \mathrm{Hg})\end{array}$ & $\begin{array}{l}{[\mathrm{Hb}]} \\
(\mathrm{g} / \mathrm{dL})\end{array}$ \\
Neutral & $266.6 \pm 35.5$ & 27.44 & 25.45 & 3.95 \\
PEG-Neutral & $189.8 \pm 20.3$ & 28.72 & 25.94 & 3.95 \\
Anionic & $151.2 \pm 17.7$ & 29.44 & 21.91 & 4.50 \\
PEG-Anionic & $135.7 \pm 5.4$ & 29.63 & 21.42 & 4.50 \\
\hline
\end{tabular}

Methemoglobin in all preparations was estimated top be $<10 \%$

The LEH-induced thrombocytopenic reaction was investigated in a rabbit model. For the purpose of following platelet circulation, the platelets were radiolabeled with In-111-oxime. The radiolabeling efficiency varied from 40-70\%. A gamma camera image of In-111-platelets circulating in a normal rabbit is shown in figure 1 . The image clearly demonstrates that the endogenous In-111-platelets circulate in blood in a stable fashion. The radioactive signal emanating from heart is indicative of blood pool.

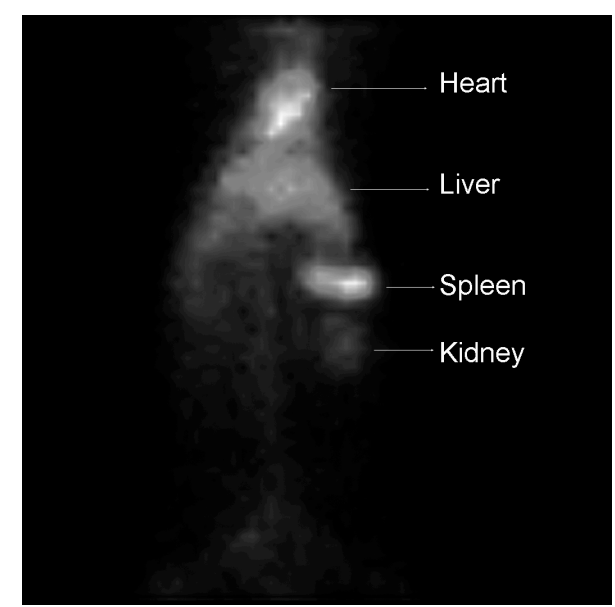

Fig. 1: A gamma camera image showing the distribution of endogenous In-111-labeled rabbit platelets in a normal rabbit $4 \mathrm{~h}$ after administration.

The radiolabeled platelets showed normal activation when stimulated with calcium and observed under a light microscope. Platelet-poor plasma was found to be the best dispersion medium for obtaining aggregate-free and homogenous suspension of radiolabeled platelets. Once injected, there was significant, but transient, sequestration of In-111platelets in RES. Since the sequestered platelets were observed to re-enter circulation within 30 minutes to provide equilibrium condition, in all cases the LEHinduced thrombocytopenic effect was studied after 30 minutes of In-111-platelets infusion.
Figure 2 shows the level of radiolabeled platelets in circulation with respect to time after LEH administration.
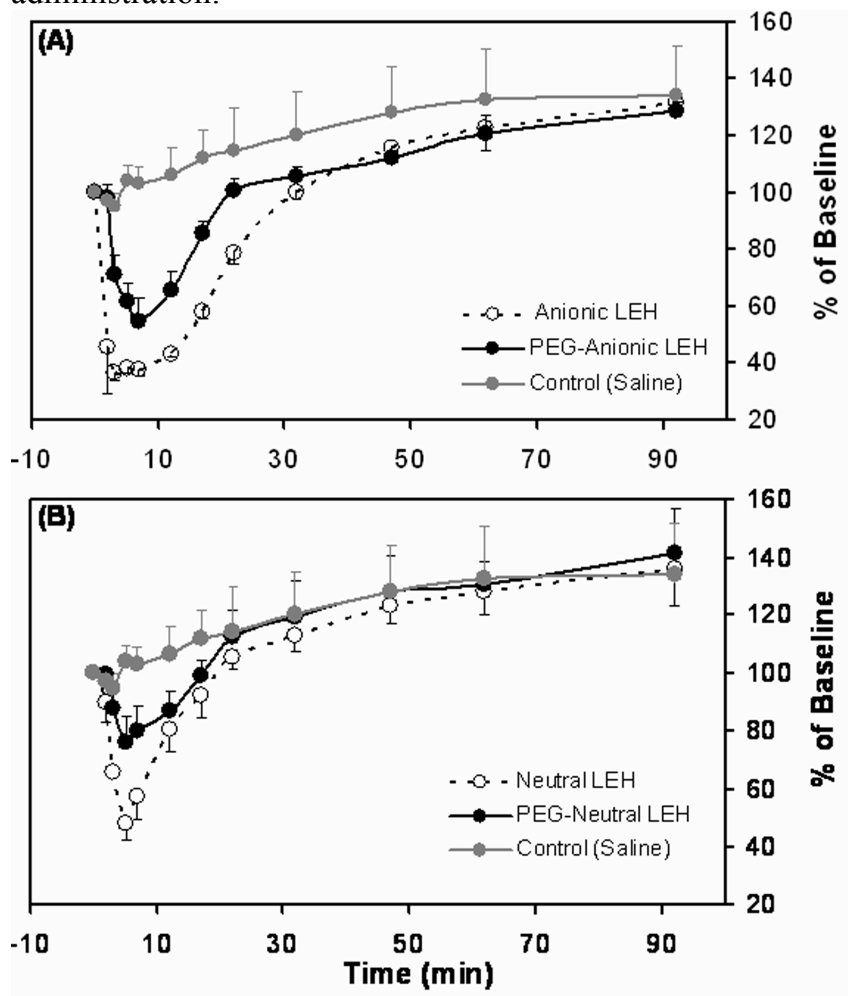

Fig. 2: Circulation kinetics of In-111-labeled platelets after administration of liposome-encapsulated liposome (LEH) preparation: (A) Anionic and PEG-Anionic LEH, and (B) Neutral and PEG-Neutral LEH.

It is clear that all LEH preparations induced a rapid and transient reduction in the circulating In-111platelets. Compared to control saline injection, the effect was very severe in the case of anionic LEH. Over $60 \%$ of In-111-platelets moved from the circulation within 5 minutes of anionic LEH injection (Figure 2A). A slight transient increase in the breathing rate was also observed in this group of animals. The radiolabeled platelets recovered in the circulation within about 30-45 minutes of anionic LEH injection. Upon PEGylation of anionic LEH, the platelet-drop was significantly reduced as compared to the anionic LEH without PEG $(p<0.05)$ and the recovery of circulating platelets was also remarkably faster $(<20 \quad \mathrm{~min})$. The apparent breathing abnormalities were also completely abrogated. In the case of neutral LEH, the decrease in circulating In-111-platelets was about 10 percentage points less than that shown by the anionic LEH (Figure 2B). The drop, however, was still larger than that seen after PEG-anionic LEH injection. When PEG-neutral 
LEH was administered, a considerably reduced thrombocytopenic effect was observed, along with a very fast recovery. Only about $20 \%$ circulating platelets dropped out of circulation after PEG-neutral LEH administration, and the recovery was observed within 10-15 minutes. No breathing abnormalities were apparent with the administration of either neutral or PEG-neutral LEH. The results clearly demonstrate that the charge carried by the liposome surface has a significant impact on the platelet reaction seen in rabbits.

Simultaneously with the withdrawal of blood for measurement of radioactivity counts in circulation, an aliquot of withdrawn anti-coagulated blood samples was also sent for automated complete blood cell counting. The circulation profile of the platelets obtained by this method (Fig. 3) corroborated very well with the data obtained from the radioactivity counting. Again, PEG-neutral LEH was the least thrombocytopenic preparation. Taken together, the results demonstrate the effectiveness of PEG-lipid in controlling an adverse thrombocytopenic reaction observed after LEH administration. The simultaneous platelet counting validated the radiolabeled platelet procedure for studying particle-induced abnormalities in circulation.

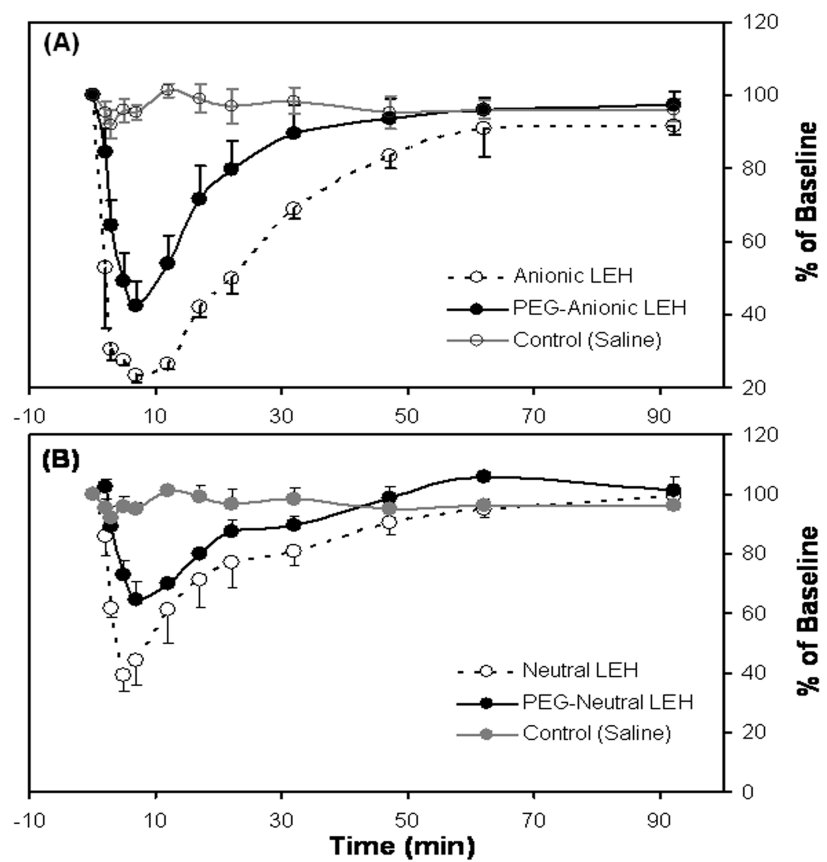

Fig. 3: Circulation kinetics of platelets after administration of LEH preparation: (A) Anionic and PEG-Anionic LEH, and (B) Neutral and PEG-Neutral LEH. Platelets were counted in blood samples by automated blood counter.
The data from circulating platelets and In-111platelets was also analyzed by plotting minima in platelet counts against the type of LEH administered (Figure 4). The partial correction of minima by a combination of neutral charge and PEGylation is emphasized yet again.

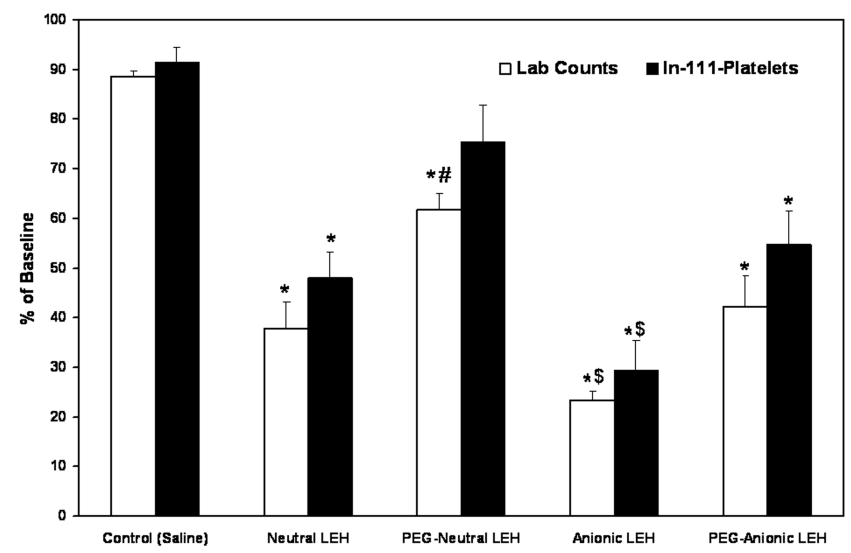

Fig. 4: The minima in circulating platelets after administration of saline (Control), Neutral, PEG-Neutral, Anionic and PEGAnionic LEH $(*$ is $\mathrm{p}<0.05$ against control, \# is $\mathrm{p}<0.05$ against Neutral, and $\$$ is $p<0.05$ against $P E G-N e u t r a l)$.

In yet another empirical observation, the thrombocytopenic effect was found to be partially influenced by the duration of LEH storage before testing in the animals. Fresh LEH preparations tested within a few days of manufacture were significantly less thrombocytopenic and better tolerated than when the preparations were tested after 2-3 months in refrigerated conditions. In the case of neutral LEH and its PEGylated version, fresh preparations were minimally thrombocytopenic. Freshly manufactured anionic LEH also demonstrated a thrombocytopenic effect, but it was of significantly less magnitude.

From the above results we conclude that PEGylation improves the circulating platelet profile of both anionic as well as neutral LEH. PEG-neutral LEH is the least thrombocytopenic formulation and therefore, is a desired composition for LEH. Moreover, storage conditions and age of the preparation have a significant impact on the apparent effect of LEH on platelets.

\section{DISCUSSION}

Liposome-encapsulated hemoglobin (LEH) is an experimental oxygen carrier that is being investigated as a resuscitation fluid in acute blood loss. It has been shown that hemorrhagic shock activates platelets 
resulting in pulmonary trapping of platelets ${ }^{[18]}$. By the very nature of its application, LEH would be used in large volumes in conditions where recipients are not only deficient in oxygen carrying capacity and circulating volume, but also in thrombogenic cellular and acellular factors. It is desirable, therefore, that any resuscitative intervention in hemorrhagic shock does not exacerbate the haemostatic imbalance. Unfortunately, foreign particles and substances that are recognized as foreign surfaces lead to platelet activation followed by their hepatic sequestration. This phenomenon has been observed in clinically used contrast agents $^{[8,}{ }^{19]}$ and may result in a prolonged clotting time

Once activated, the platelets secrete potent selfactivating substances: ADP, PAF, etc., which synergistically potentiate the effects of primary activators. Apparently, the particle-induced platelet activation is different from the activation induced by soluble activators, such as thrombin ${ }^{[8]}$. Thrombininduced platelet activation does not cause protein tyrosine phosphorylation and is dependent on intracellular cyclic adenosine monophosphate $\left(\mathrm{cAMP}_{\mathrm{i}}\right)$. On the other hand, particulate agonists activate platelets following tyrosine phosphorylation of proteins, and the

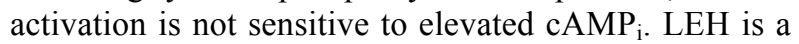
particulate formulation, but it has not been investigated in this regard. The general nature of particle-induced platelet activation suggests a similar mechanism for LEH-induced platelet activation. Presence of free hemoglobin in preparations of LEH may compound the problem, because decreased cGMP levels through the depletion of $\mathrm{NO}$ can lead to platelet activation and aggregation $^{[20]}$.

Human platelets have the capacity of actually phagocytosing the liposomes ${ }^{[21]}$. The transient nature of liposome-platelet interaction in rabbits was first reported by Doerschuk, et al. ${ }^{[22]}$. The investigators found that while phosphatidylglycerol liposomes produced $41 \%$ reduction in circulating platelets, neutral egg phosphatidylcholine liposomes did not affect circulating platelets. In a subsequent in vitro study, the authors suggested the role of complement proteins in phosphatidylglycerol liposome-platelet interaction ${ }^{[10]}$. Early during the development of LEH, intravenous administration of LEH was found to cause several transient inadvertent effects characterized by hypertension, tachycardia, thrombocytopenia, hemoconcentration and elevation of plasma thromboxane B2. These reactions obliterated when platelet-activating factor antagonist BN 50739 was coadministered $^{[23]}$. The role of complement in LEHinduced thrombocytopenia was yet again reported by
Goins, et al ${ }^{[5]}$. Complement depleted rats were unable to show any reduction in circulating platelets after LEH administration $^{[5]}$. In the same year, it was reported that the LEH-induced TXB2 response in rats was inhibited by co-administration of soluble $\mathrm{C}$ receptor type 1 (sCR1), providing further evidence for a causal relationship between LEH-induced $\mathrm{C}$ activation and TXB2 release ${ }^{[24]}$. These studies support the hypothesis that complement activation is pivotal to and precedes LEH-induced thrombocytopenia.

While the 'how' question seems to have been addressed, what in LEH causes thrombocytopenia is still not clear. Several factors might play a role in this phenomenon, including liposome size, presence of trace free hemoglobin, charge and overall composition of lipid structure. Surface modification of LEH with hydrophilic polymers to prepare 'stealth' LEH has been found to reduce LEH-platelet interaction. For instance, Wakamoto, et al., investigated the ability of PEGmodified LEH to release RANTES (regulated upon activation, normal T-cell expressed and presumably secreted) from platelets and found that PEG-coated LEH ( $2 \%$ concentration) has no effect on platelet function in vitro ${ }^{[25]}$. More recently, in a similar study, the same group of investigators reported that the hemoglobin lipid vesicles do not have any adverse effect even at $40 \%$ concentration ${ }^{[26]}$. In this work, we studied in vivo platelet activation and demonstrated the utility of PEGylation in alleviating the thrombocytopenic response induced by our formulation of LEH. We also showed that the magnitude of acute thrombocytopenia is dependent on the charge carried by the liposomes. Anionic LEH showed massive relocation in circulating platelets. A few animals were imaged by gamma camera for visual assessment of platelet distribution after LEH administration (data not shown). It was found that the majority of platelets were sequestered by the liver followed by moderate to minor accumulation in lung and spleen. The images also show gradual re-population of platelets in circulation as observed by actual counting of platelets. Since we report that the thrombocytopenic effect is transient and rapid in nature, any investigation on activation of platelets after about 30 minutes of liposome administration will be of limited value.

Apart from reducing platelet interaction, PEGylation of LEH formulation adds several other valuable properties. LEH composed of phosphatidylcholine/cholesterol is rapidly eliminated from the body by the RES. Often the elimination process is preceded by interaction of particulate LEH with complement and other opsonizing proteins resulting in a form of 
pseudoallergic reactions described as complement activation-related pseudoallergy or CARPA ${ }^{[27]}$. PEGylation conceals the liposome surface by steric modification. It is believed that a hydrophilic coating on the liposome surface creates a steric barrier, enabling liposomes to circulate longer ${ }^{[11]}$. The other benefits of PEG-modification are the reduction of particle aggregation and modification of LEH viscosity. These effects improve the flow properties of LEH through narrow capillaries ${ }^{[28]}$. A conventional way of liposome PEGylation is to add PEG-lipid in the lipid phase just prior to its hydration with an aqueous phase $^{[29]}$. However, this technique results in the PEG brush or mushroom occupying the limited space inside the liposomes. The same steric hindrance that makes PEG useful may inhibit the encapsulation of substances. The smaller the size of the liposomes, the greater is the impact of PEG on total usable space for encapsulated material. Since PEG-lipid associated with the outer lipid layer is useful for effective stealthing, the conventional method of PEGylation requires more PEG-lipid than is needed. In the case of multi-lamellar liposomes, the magnitude of PEG-PE wastage is more. Realization of the problems associated with conventional PEGylation led to the development of a technique where PEG-PE is inserted in the outer layer of liposomes after the final manufacturing stages ${ }^{[30,31]}$. This technique, called post-insertion, is especially useful in the case of LEH. Post-insertion technique not only doubles the circulation $\mathrm{T}_{1 / 2}$ of $\mathrm{LEH}$, it also improves the encapsulation efficiency of hemoglobin ${ }^{[12,}$ 32].

In conclusion, we report that an LEH formulation composed of neutral lipids and modified by postinsertion of PEG-lipid, helps in reduction of thrombocytopenic reaction. It would be of interest to further investigate the role of polymer chain length (PEG5000 versus PEG 2000) and the type of lipid anchor in this regard.

\section{ACKNOWLEDGEMENTS}

The authors acknowledge Dr. Alan Rudolph's and Mr. Richard Cliff's help in providing us with human stroma-free hemoglobin from US Army Medical Research and Development Command, Washington DC. We also thank Miss Delia Garcia and Mr. Robert Klipper for the technical help. This work was supported by a grant (Award number N00140010793) from the Office of Naval Research, Washington DC (USA).

\section{REFERENCES}

1. Awasthi V.D., Garcia D., Klipper R., Phillips W.T., and Goins B.A., 2004. Kinetics of liposomeencapsulated hemoglobin after $25 \%$ hypovolemic exchange transfusion. Int. J. Pharm., 283:53-62.

2. Sakai H., Yuasa M., Onuma H., Takeoka S., and Tsuchida E., 2000. Synthesis and physicochemical characterization of a series of hemoglobin-based oxygen carriers: Objective comparison between cellular and acellular types. Bioconjugate Chem., 11 56-64.

3. Phillips W.T., Klipper R., Rudolph A.S., and Goins B., 1994. Investigation of liposome-encapsulated hemoglobin/platelet interaction using indium-111 labeled platelets. Artif. Cells Blood Subs. Immob. Biotechnol., 22 A144.

4. Phillips W.T., Klipper R., Fresne D., Rudolph A.S., Javors M., and Goins B., 1997. Platelet reactivity with liposome-encapsulated hemoglobin in the rat. Exp. Hematol., 25: 1347-1356.

5. Goins B., Phillips W.T., Klipper R., and Rudolph A.S., 1997. Role of complement in rats injected with liposome-encapsulated hemoglobin. J. Surg. Res., 68: 99-105.

6. Bruni A., and Palatini P., 1982. Biological and pharmacological properties of phospholipids. Prog. Med. Chem., 19: 111-203.

7. Yasuda T., Tadakuma T., Pierce C.W., and Kinsky S.C., 1979. Primary in vitro immunogenicity of liposomal model membranes in mouse spleen cell cultures. J. Immunol., 123: 1535-1539.

8. Ryningen A., and Holmsen H., 1998. Role of autocrine stimulation for the effects of cyclic AMP on protein and lipid phosphorylation in platelets activated by particles. FEBS Lett., 436: 335-338.

9. Moghimi S.M., Hamad I., Andresen T.L., Jorgensen K., and Szebeni J., 2006. Methylation of the phosphate oxygen moiety of phospholipidmethoxy(polyethylene glycol) conjugate prevents PEGylated liposome-mediated complement activation and anaphylatoxin production. FASEB J., 20: 2591-2593.

10. Loughrey H.C., Bally M.B., Reinish L.W., and Cullis P.R., 1990. The binding of phosphatidylglycerol liposomes to rat platelets is mediated by complement. Thromb. Haemost., 64: 172-176.

11. Torchilin V.P., and Papisov M.I., 1994. Why do polyethylene glycol-coated liposomes circulate so long? J. Liposome Res., 4: 725-739.

12. Awasthi V.D., Garcia D., Klipper R., Goins B.A., and Phillips W.T., 2004. Neutral and anionic liposome-encapsulated hemoglobin: Effect of postinserted poly (ethylene glycol)distearoylphosphatidylethanolamine on distribution and circulation Kinetics. J. Pharmacol. Exp. Ther., 309: 241-248. 
13. Sakai H., Takeoka S., Park S.I., Kose T., Nishide H., Izumi Y., Yoshizu A., Kobayashi K., and Tsuchida E., 1997. Surface modification of hemoglobin vesciles with poly(ethylene glycol) and effects on aggregation, viscosity, and blood flow during $90 \%$ exchange transfusion in anesthetized rats. Bioconjugate Chem., 8: 23-30.

14. Stewart J.C.M., 1980. Colorimetric determination of phospholipids with ammonium ferrothiocyanate. Anal. Biochem., 104: 10-14.

15. Tomita S., Enoki Y., Santa M., Yoshida H., and Yasumitsu Y., 1968. A simple spectrophotometric method for determination of met-hemoglobin in dilute solution. J. Nara. Med. Assoc., 19: 1-6.

16. Matsuoka T., 1997. Determination of methemoglobin and carboxyhemoglobin in blood by rapid colorimetry. Biol Pharm Bull, 20 (11): 1208-1211.

17. Tsan M.F., and Hill-Zobel R.L., 1987. Should platelets be radiolabelled in plasma medium? Am. J. Hematol., 25: 355-359.

18. Peer R.M., and Schwartz S.I., 1975. Development and treatment of post-traumatic pulmonary platelet trapping. Ann. Surg., 181: 447-451.

19. Szebeni J., 2004. Hypersensitivity reactions to radiocontrast media: the role of complement activation. Curr. Allergy Asthma Rep., 4: 25-30.

20. Rother R.P., Bell L., Hillmen P., and Gladwin M.T., 2005. The clinical sequelae of intravascular hemolysis and extracellular plasma hemoglobin: a novel mechanism of human disease. JAMA, 293: 1653-1662.

21. Male R., Vannier W.E., and Baldeschwieler J.D., 1992. Phagocytosis of liposomes by human platelets. Proc. Natl. Acad. Sci. USA, 89: 91919195.

22. Doerschuk C.M., Gie R.P., Bally M.B., Cullis P.R., and Reinish L.W., 1989. Platelet distribution in rabbits following infusion of liposomes. Thromb. Haemost., 61: 392-396.

23. Rabinovici R., Rudolph A.S., Yue T.L., and Feuerstein G., 1990. Biological responses to liposome-encapsulated hemoglobin (LEH) are improved by a PAF antagonist. Circ. Shock, 31: 431-445.

24. Szebeni J., Spielberg H., Cliff R.O., Wassef N.M., Rudolph A.S., and Alving C.R., 1997. Complement activation and thromboxane secretion by liposomeencapsulated hemoglobin in rats in vivo: inhibition by soluble complement receptor type 1. Artif. Cells Blood Subs. Immob. Biotechnol., 25: 347-355.
25. Wakamoto S., Fujihara M., Abe H., Sakai H., Takeoka S., Tsuchida E., Ikeda H., and Ikebuchi K., 2001. Effects of poly(ethyleneglycol)-modified hemoglobin vesicles on agonist-induced platelet aggregation and RANTES release in vitro. Artif. Cells Blood Subs. Immob. Biotechnol., 29: 191201.

26. Wakamoto S., Fujihara M., Abe H., Yamaguchi M., Azuma H., Ikeda H., Takeoka S., and Tsuchida E., 2005. Effects of hemoglobin vesicles on resting and agonist-stimulated human platelets in vitro. Artif. Cells Blood Subs. Immob. Biotechnol., 33: 101-111.

27. Szebeni J., Baranyi L., Savay S., Bodo M., Morse D.S., Basta M., Stahl G.L., Bunger R., and Alving C.R., 2000. Liposome-induced pulmonary hypertension: properties and mechanism of a complement-mediated pseudoallergic reaction. Am. J. Physiol., 279: H1319-1328.

28. Sakai H., Takeoka S., Park S.I., Kose T., Nishide H., Izumi Y., Yoshizu A., Kobayashi K., and Tsuchida E., 1997. Surface modification of hemoglobin vesicles with poly(ethylene glycol) and effects on aggregation, viscosity, and blood flow during $90 \%$ exchange transfusion in anesthetized rats. Bioconjugate Chem., 8: 23-30.

29. Phillips W.T., Klipper R.W., Awasthi V.D., Rudolph A.S., Cliff R., Kwasiborski V., and Goins B.A., 1999. Polyethylene glycol-modified liposome-encapsulated hemoglobin: a long circulating red cell substitute. The J. Pharmacol. Exp. Ther., 288: 665-670.

30. Sou K., Endo T., Takeoka S., and Tsuchida E., 2000. Poly(ethylene glycol)-modification of the phospholipid vesicles by using the spontaneous incorporation of poly(ethylene glycol)-lipid into the vesicles. Bioconjugate Chem., 11: 372-379.

31. Uster P.S., Allen T.M., Daniel B.E., Mendez C.J., Newman M.S., and Zhu G.Z., 1996. Insertion of poly(ethylene glycol) derivatized phospholipid into pre-formed liposomes results in prolonged in vivo circulation time. FEBS Lett., 386: 243-246.

32. Sou K., Naito Y., Endo T., Takeoka S., and Tsuchida E., 2003. Effective encapsulation of proteins into size-controlled phospholipid vesicles using freeze-thawing and extrusion. Biotechnol. Prog., 19: 1547-1552. 